\title{
Math-Net.Ru
}

Н. И. Казимиров, О некоторых условиях отсутствия гигантской компоненты в обобщенной схеме размещения, Дискрет. матем., 2002, том 14, выпуск 2, 107-118

DOI: https://doi.org/10.4213/dm245

Использование Общероссийского математического портала Math-Net.Ru подразумевает, что вы прочитали и согласны с пользовательским соглашением http://www.mathnet.ru/rus/agreement

Параметры загрузки:

IP : 54.224 .187 .69

26 апреля 2023 г., 14:51:47

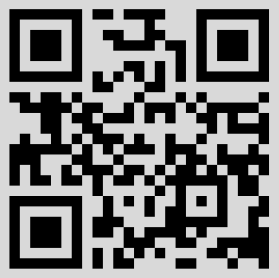




\title{
О некоторых условиях отсутствия гигантской компоненты в обобщенной схеме размещения
}

\author{
() 2002 г. Н. И. Казимиров
}

Доказаны две теоремы, которые содержат условия отсутствия гигантской компоненты в обобщенной схеме размещения. Приведены примеры применения этих теорем.

Работа выполнена при поддержке Российского фонда фундаментальных исследований, проект 00-01-00233.

Для решения многих комбинаторных задач часто используется вероятностный подход. Если на множестве изучаемых комбинаторных объектов задать вероятностную меру, выбранную в соответствии с конкретными прикладными целями, то числовые характеристики таких объектов можно рассматривать как случайные величины. Множество примеров применения такого подхода можно найти в $[1,2,3]$, где одним из главных методов исследования является обобщенная схема размещения частиц по ячейкам [1].

Пусть имеется набор целочисленных неотрицательных случайных величин

$$
\eta_{1}, \ldots, \eta_{N}
$$

таких, что $\eta_{1}+\ldots+\eta_{N}=n$ и совместное распределение $\eta_{1}, \ldots, \eta_{N}$ задано с помощью последовательности $b=\left(b_{0}, b_{1}, \ldots\right)$ неотрицательных чисел следующим образом:

$$
\mathbf{P}\left\{\eta_{1}=n_{1}, \ldots, \eta_{N}=n_{N}\right\}=\frac{b_{n_{1}} \ldots b_{n_{N}}}{\sum_{i_{1}+\ldots+i_{N}=n} b_{i_{1}} \ldots b_{i_{N}}} .
$$

Введем обозначение

$$
B(x)=\sum_{k} b_{k} x^{k}
$$

Пусть последовательность $b$ такова, что радиус сходимости $B(x)$ отличен от нуля. Пусть случайные величины $\xi_{1}, \xi_{2}, \ldots$ независимы, одинаково распределены и

$$
\mathbf{P}\left\{\xi_{1}=k\right\}=\frac{b_{k} x^{k}}{B(x)}
$$

Тогда, как легко видеть,

$$
\mathbf{P}\left\{\eta_{1}=n_{1}, \ldots, \eta_{N}=n_{N}\right\}=\mathbf{P}\left\{\xi_{1}=n_{1}, \ldots, \xi_{N}=n_{N} \mid \xi_{1}+\ldots+\xi_{N}=n\right\} .
$$

В этом случае мы говорим, что наборы случайных величин $\eta_{1}, \ldots, \eta_{N}$ и $\xi_{1}, \ldots, \xi_{N}$ образуют обобщенную схему размещения. 
В последнее время повысился интерес к исследованию обобщенной схемы размещения с точки зрения возникновения в ней гигантской компоненты [4]. Пусть

$$
\eta_{(1)} \leqslant \eta_{(2)} \leqslant \ldots \leqslant \eta_{(N)}
$$

- вариационный ряд случайных величин $\eta_{1}, \ldots, \eta_{N}$. Говорят, что в обобщенной схеме размещения возникает гигантская компонента, если при $N, n \rightarrow \infty$ величина $\eta_{(N)}$ с вероятностью, стремящейся к единице, имеет порядок $n$, и случайные величины $\eta_{(N)}$ и $\eta_{(N-1)}$ имеют предельные распределения при различной по порядку нормировке. В [4] приведено несколько примеров обобщенной схемы размещения как с гигантской компонентой, так и без нее. Следует отметить, что вопрос об общем виде распределения $\xi_{1}$ для схем с гигантской компонентой остается открытым.

Изучение вероятностей вида (1) комбинаторными методами часто бывает затруднительно, однако представление их в виде (3) позволяет находить их асимптотику при $N, n \rightarrow \infty$ с помощью хорошо разработанного аппарата предельных теорем теории вероятностей. Кроме того, инвариантность вероятностей (3) относительно параметра $x$ позволяет осуществить его выбор (в зависимости от $N, n$ ) способом, наиболее удобным для получения предельных теорем.

Ниже доказаны теоремы 1 и 2, которые дают некоторые простые достаточные условия невозникновения гигантской компоненты в обобщенной схеме размещения. Доказательства этих теорем основаны на леммах 3 и 4 о локальной сходимости для сумм независимых случайных величин в схеме серий. Следует отметить, что в задачах, сводимых к обобщенной схеме размещения $[1,3]$, получение именно такого рода результатов представляет наибольшие трудности.

Пусть $k_{0}$ - наименьшее число такое, что $b_{k_{0}}>0$. Мы не рассматриваем здесь случай вырожденного распределения $\xi_{1}$, поэтому по меньшей мере два элемента последовательности $b$ положительны. Обозначим через $d$ максимальный шаг распределения $\xi_{1}$. Тогда случайная величина $\left(\xi_{1}-k_{0}\right) / d$ принимает нулевое значение с положительной вероятностью, и максимальный шаг ее распределения равен 1. Поэтому далее, не теряя общности, мы предполагаем, что $b_{0}>0$ и $d=1$.

Введем необходимые обозначения. Пусть $R$ - радиус сходимости $B(x)$. Как уже отмечалось выше, предполагается, что $R>0$. Пусть $|b|=\left\{0, k_{1}, k_{2}, \ldots\right\}$ - носитель последовательности $b$, где $0<k_{1}<k_{2}<\ldots$ Тогда $\operatorname{gcd}|b|=d=1$, где gcd означает наибольший общий делитель. Пусть $s-$ наименьшее число такое, что $\operatorname{gcd}\left\{k_{1}, \ldots, k_{s}\right\}=1, m=k_{s}$. Рассмотрим независимые одинаково распределенные случайные величины $\zeta_{1}, \ldots, \zeta_{N}$ такие, чTO

$$
\mathbf{P}\left\{\zeta_{1}=k\right\}=\mathbf{P}\left\{\xi_{1}=k \mid \xi_{1} \leqslant r\right\}, \quad k \leqslant r .
$$

\section{Положим также}

$$
\begin{aligned}
P_{r}=\mathbf{P}\left\{\xi_{1}>r\right\}, \quad a=\mathbf{E} \xi_{1}, & \sigma^{2}=\mathbf{D} \xi_{1}, \quad \mu_{4}=\mathbf{E}\left(\xi_{1}-a\right)^{4} \\
S_{N} & =\xi_{1}+\ldots+\xi_{N},
\end{aligned}
$$

Если $X$ - случайная величина, то $\varphi(X ; t)$ обозначает ее характеристическую функцию $E \exp \{i t X\}$. При выводе асимптотических соотношений удобно пользоваться следующими обозначениями [5]: если для некоторых двух (действительных) функций $v(y)$ и $w(y)$, определенных на множестве $Y$ с предельной точкой $y_{0},\left|y_{0}\right| \leqslant \infty$, при $y \rightarrow y_{0}$ выполнено соотношение $v(y)=O(w(y))$, то мы пишем $v(y) \preccurlyeq w(y)$ или $w(y) \succcurlyeq v(y)$; если имеют 
место соотношения $v(y) \preccurlyeq w(y)$ и $v(y) \succcurlyeq w(y)$ одновременно, то будем писать $v(y) \asymp$ $w(y)$.

Нетрудно проверить (см. $[1,2,3])$, что

$$
\mathbf{P}\left\{\eta_{(N)} \leqslant r\right\}=\left(1-P_{r}\right)^{N} \frac{\mathbf{P}\left\{Z_{N}=n\right\}}{\mathbf{P}\left\{S_{N}=n\right\}} .
$$

Отсюда видно, что изучение свойств максимальной компоненты обобщенной схемы размещения можно свести к изучению полинома $\left(1-P_{r}\right)^{N}$ и сумм $S_{N}$ и $Z_{N}$.

Отметим, что мы не рассматриваем здесь вариант $R=\infty, \sigma \rightarrow \infty$, поскольку из всех широко известных примеров этим условиям удовлетворяют только задачи, в которых (2) является распределением Пуассона (классическая схема размещения) с неограниченно растущим параметром или урезанным распределением Пуассона (случайные неупорядоченные разбиения конечного множества на непересекающиеся подмножества). Для таких задач отсутствие гигантской компоненты установлено в $[2,3]$.

Для начала изучим вопрос о слабой сходимости сумм $S_{N}$ и $Z_{N}$. Следующая лемма дает возможность получать предельные распределения сумм $Z_{N}$ усеченных слагаемых, если известно предельное распределение $S_{N}$.

Лемма 1. Пусть $N \rightarrow \infty, r$ выбрано так, что $N P_{r} \rightarrow 0$. Тогда при любом фиксированном $t$

$$
\varphi\left(\left(S_{N}-N a\right) /(\sigma \sqrt{N}), t\right) / \varphi\left(\left(Z_{N}-N a\right) /(\sigma \sqrt{N}), t\right) \rightarrow 1
$$

Доказательство. Раскладывая $\varphi\left(\xi_{1}-a, u\right)$ по формуле Тейлора по переменной $и$ в окрестности нуля до второй производной, получим, что

$$
\varphi\left(\xi_{1}-a, u\right)=1-\frac{u^{2}}{2} \mathbf{E}\left(\xi_{1}-a\right)^{2} e^{i \theta\left(\xi_{1}-a\right)}, \quad 0 \leqslant|\theta| \leqslant|u| .
$$

Поскольку $\left|\mathbf{E}\left(\xi_{1}-a\right)^{2} e^{i \theta\left(\xi_{1}-a\right)}\right| \leqslant \sigma^{2}$, при каждом фиксированном $t$

$$
\varphi\left(\xi_{1}-a, t /(\sigma \sqrt{N})\right) \rightarrow 1
$$

Далеe,

$$
\begin{aligned}
\varphi\left(\frac{Z_{N}-N a}{\sigma \sqrt{N}}, t\right)=\varphi\left(\frac{S_{N}-N a}{\sigma \sqrt{N}}\right. & , t)\left(1-P_{r}\right)^{-N} \\
& \times\left(1-\frac{1}{\varphi\left(\xi_{1}-a, t /(\sigma \sqrt{N})\right)} \sum_{k>r} e^{i t k} \mathbf{P}\left\{\xi_{1}=k\right\}\right)^{N} .
\end{aligned}
$$

Поскольку $N P_{r} \rightarrow 0$, очевидно, что $\left(1-P_{r}\right)^{N} \rightarrow 1$ и

$$
N\left|\sum_{k>r} e^{i t k} \frac{\mathbf{P}\left\{\xi_{1}=k\right\}}{\varphi\left(\xi_{1}-a, t /(\sigma \sqrt{N})\right)}\right| \leqslant \frac{N P_{r}}{\left|\varphi\left(\xi_{1}-a, t /(\sigma \sqrt{N})\right)\right|} \rightarrow 0 .
$$

Отсюда и из (7) следует, что последний сомножитель в правой части (8) также стремится к 1. Тем самым мы получаем соотношение (6). 
Лемма 2. Пусть $N \rightarrow \infty, r \asymp N a$ и выполнено одно из условий:

$(\alpha) \sigma \sqrt{N} \rightarrow \infty, \mathbf{E} \xi_{1}^{2} \preccurlyeq 1$,

(в) $\mu_{4} /\left(\sigma^{4} N\right) \rightarrow 0$.

Тогда

$$
\varphi\left(\frac{S_{N}-N a}{\sigma \sqrt{N}}, t\right) \rightarrow e^{-t^{2} / 2}, \quad \varphi\left(\frac{Z_{N}-N a}{\sigma \sqrt{N}}, t\right) \rightarrow e^{-t^{2} / 2}
$$

$u \sigma \sqrt{N}=o(N a)$.

Доказательство. По теореме Линдеберга для доказательства слабой сходимости $\left(S_{N}-N a\right) /(\sigma \sqrt{N})$ к нормальному закону достаточно показать, что для любого $\tau>0$

$$
L_{N}(\tau)=\sum_{k=1}^{N} \mathbf{E}\left(\frac{\left(\xi_{k}-a\right)^{2}}{N \sigma^{2}}, \frac{\left|\xi_{k}-a\right|}{\sigma \sqrt{N}}>\tau\right) \rightarrow 0
$$

Для $L_{N}(\tau)$ справедливы оценки

$$
L_{N}(\tau)=\frac{N}{N \sigma^{2}} \sum_{|k-a|>\tau \sigma \sqrt{N}}(k-a)^{2} \mathbf{P}\left\{\xi_{1}=k\right\} \leqslant \sigma^{-2} \sum_{|k-a|>\tau \sigma \sqrt{N}} k^{2} \mathbf{P}\left\{\xi_{1}=k\right\}
$$

и, аналогично,

$$
L_{N}(\tau) \leqslant \sigma^{-2} \mu_{4} /\left(\tau^{2} \sigma^{2} N\right) .
$$

Если выполнено условие $(\alpha)$, то $a \preccurlyeq 1$ и, учитывая условие $\sigma \sqrt{N} \rightarrow \infty$, получаем, что $L_{N}(\tau) \rightarrow 0$. Если выполнено условие $(\beta)$, воспользуемся второй оценкой. Таким образом, $\left(S_{N}-N a\right) /(\sigma \sqrt{N})$ слабо сходится к нормальному закону, и мы получаем первое из соотношений (9).

Докажем, что $\sigma \sqrt{N}=o(N a)$. Из слабой сходимости $\left(S_{N}-N a\right) /(\sigma \sqrt{N})$ к нормальному закону следует, что для любого $t>0$

$$
\mathbf{P}\left\{S_{N}<-t \sigma \sqrt{N}+N a\right\} \rightarrow \frac{1}{\sqrt{2 \pi}} \int_{-\infty}^{-t} e^{-u^{2} / 2} d u=\gamma_{t}
$$

Предположим, что $N a \leqslant c \sigma \sqrt{N}$, где постоянная $c>0$. Тогда

$$
\mathbf{P}\left\{S_{N}<-(c+1) \sigma \sqrt{N}+N a\right\} \leqslant \mathbf{P}\left\{S_{N}<-\sigma \sqrt{N}\right\}=0,
$$

что противоречит тому, что $\gamma_{c+1}>0$. Следовательно, $\sigma \sqrt{N}=o(N a)$.

Отсюда и из того, что $a=o(r)$, поскольку $r \asymp N a$, получаем оценку для $N P_{r}$ :

$$
N P_{r}=N \mathbf{P}\left\{\xi_{1}>r\right\} \leqslant N \mathbf{P}\left\{\left|\xi_{1}-a\right|>r / 2\right\} \preccurlyeq N \sigma^{2} / r^{2} \rightarrow 0 .
$$

Отсюда по лемме 1 получаем второе из соотношений (9).

В классической теореме Линдеберга в качестве центрирующих и нормирующих коэффициентов слагаемых выбираются, соответственно, математическое ожидание и дисперсия этих же слагаемых. Поэтому мы не можем непосредственно воспользоваться этой теоремой для получения второго из соотношений (9), так как, вообще говоря, равенства $\mathbf{E} Z_{N}=N a, \mathbf{D} Z_{N}=N \sigma^{2}$ не верны. Лемма 1 позволяет в ряде случаев обойти данное ограничение.

Рассмотрим теперь вопрос о локальной сходимости распределений $S_{N}$ и $Z_{N}$. 
Лемма 3. Пусть $N \rightarrow \infty, N x^{m} \rightarrow \infty, \mathbf{E} \xi_{1}^{2} \preccurlyeq 1, r \asymp N a$. Тогда равномерно относительно иельх $h$

$$
\sigma \sqrt{N} \mathbf{P}\left\{Z_{N}=h\right\}-\frac{1}{\sqrt{2 \pi}} \exp \left\{-\frac{(h-N a)^{2}}{2 \sigma^{2} N}\right\} \rightarrow 0 .
$$

Доказательство. Из условия $N x^{m} \rightarrow \infty$ следует, что $N x^{k_{1}} \rightarrow \infty$. Кроме того, $\sigma^{2} \asymp x^{k_{1}}$. Действительно, из условия $\mathbf{E}_{1}^{2} \preccurlyeq 1$ получаем, что $B(x) \asymp 1, x \preccurlyeq 1$. Если $x \asymp 1$, то $a \asymp 1$, откуда $\sigma^{2} \geqslant a^{2} b_{0} / B(x) \asymp 1$. Если же $x \rightarrow 0$, то $a \asymp x^{k_{1}}, \mathbf{E} \xi_{1}^{2} \asymp x^{k_{1}}$, откуда $\sigma^{2} \asymp x^{k_{1}}$.

Таким образом, из условия $N x^{m} \rightarrow \infty$ следует, что $N \sigma^{2} \rightarrow \infty$. Поэтому в условиях доказываемой леммы выполняются условия леммы 2 , так как при $x \geqslant \varepsilon$, где $\varepsilon-$ произвольно малое положительное число, $\mathbf{E} \xi_{1}^{2} \asymp 1$ и $\sigma \sqrt{N} \rightarrow \infty$, а при $x \rightarrow 0$ аналогично предыдущему получаем, что $\mathbf{E}\left(\xi_{1}-a\right)^{p} \asymp x^{k_{1}}, p \geqslant 2$, откуда $\mu_{4} /\left(\sigma^{4} N\right) \asymp\left(N x^{k_{1}}\right)^{-1} \rightarrow 0$. Следовательно, $Z_{N}$ слабо сходится к нормальному закону.

Пусть

$$
\begin{aligned}
H_{N} & =N \inf _{1 / 4 \leqslant t \leqslant 1 / 2} \mathbf{E}\left\langle\left(\zeta_{1}-\zeta_{2}\right) t\right\rangle^{2}, \\
E_{N}^{2}(u) & =2 N \sum_{1 \leqslant k \leqslant u} k^{2} \mathbf{P}\left\{\zeta_{1}-\zeta_{2}=k\right\},
\end{aligned}
$$

где $\langle y\rangle$ - расстояние от $y$ до ближайшего целого числа. По теореме 1 из [6] для того, чтобы показать, что в условиях данной леммы из интегральной сходимости $Z_{N}$ следует локальная, достаточно проверить, что $H_{N} \rightarrow \infty$ и $\sigma \sqrt{N} \preccurlyeq E_{N}(u)$ при некотором фиксированном $u>0$.

Докажем, что для любого $t \in[1 / 4,1 / 2]$ существует $k_{j}, 1 \leqslant j \leqslant s$, такое, что $\left\langle k_{j} t\right\rangle>0$. Предположим, что при некотором $t$ для всех $k_{j}$ число $k_{j} t$ целое. Отсюда, в частности, следует, что $t$ рационально. Тогда $t=l_{1} / l_{2}$, где $l_{1}, l_{2}$ - целые положительные взаимно простые числа. При этом из неравенства $t \leqslant 1 / 2$ следует, что $l_{2} \geqslant 2$. Для любого $j=$ $1, \ldots, s$ число $k_{j} l_{1}$ кратно $l_{2}, \operatorname{gcd}\left\{l_{1}, l_{2}\right\}=1$, откуда все $k_{j}$ кратны $l_{2}$, что противоречит условию $\operatorname{gcd}\left\{k_{1}, \ldots, k_{s}\right\}=1$.

Из доказанного следует, что при $t \in[1 / 4,1 / 2]$

$$
f(t)=\sum_{j=1}^{s}\left\langle k_{j} t\right\rangle^{2}>0 .
$$

Кроме того, $f(t)$ непрерывна, поэтому

$$
\inf _{1 / 4 \leqslant t \leqslant 1 / 2} f(t)>0
$$

Отсюда получаем, что

$$
\begin{aligned}
\mathbf{E}\left\langle\left(\zeta_{1}-\zeta_{2}\right) t\right\rangle^{2} & =\sum_{i \in \mathbf{Z}}\langle i t\rangle^{2} \sum_{k=\max \{0,-i\}}^{\min \{r, r-i\}} \frac{b_{k} b_{k+i} x^{2 k+i}}{B^{2}(x)\left(1-P_{r}\right)^{2}} \\
& \geqslant \sum_{j=1}^{s}\left\langle k_{j} t\right\rangle^{2} \frac{b_{0} b_{k_{j}} x^{k_{j}}}{B^{2}(x)\left(1-P_{r}\right)^{2}} \succcurlyeq \sum_{j=1}^{s}\left\langle k_{j} t\right\rangle^{2} x^{k_{j}} \succcurlyeq x^{m} .
\end{aligned}
$$

Отсюда и из условия $N x^{m} \rightarrow \infty$ следует, что $H_{N} \rightarrow \infty$. 
Оценим $E_{N}^{2}\left(k_{1}\right)$. Нетрудно видеть, что

$$
\begin{aligned}
E_{N}^{2}\left(k_{1}\right) & =2 N \sum_{k=1}^{k_{1}} k^{2} \sum_{i=0}^{r-k_{1}} b_{i} b_{i+k} x^{2 i+k} /\left(B(x)\left(1-P_{r}\right)\right)^{2} \\
& \geqslant 2 N k_{1}^{2} b_{0} b_{k_{1}} x^{k_{1}} /\left(B(x)\left(1-P_{r}\right)\right)^{2} \succcurlyeq N x^{k_{1}}
\end{aligned}
$$

Отсюда и из соотношения $\sigma^{2} \preccurlyeq x^{k_{1}}$ получаем, что $\sigma \sqrt{N} \preccurlyeq E_{N}\left(k_{1}\right)$. Лемма доказана.

Заметим, что если при доказательстве леммы 3 всюду считать $r=\infty$, то мы получим, что в условиях этой леммы выполнена локальная сходимость распределения $S_{N}$ к нормальному распределению.

Как видно из условий леммы 3, ограниченность второго момента $\xi_{1}$ позволяет доказать локальную сходимость $S_{N}$ для достаточно широкого класса последовательностей $b$, так как мы здесь не требуем какой-то конкретной асимптотики $b_{k}$ при $k \rightarrow \infty$. Однако снятие условия ограниченности второго момента $\xi_{1}$ приводит к более жестким ограничениям на вид $b_{k}$.

Ниже мы рассмотрим случай $b_{k} \asymp k^{-\delta}$ при $k \rightarrow \infty, 1<\delta<3$. Отметим, что если $\delta>3$, то второй момент $b$ конечен, и эта ситуация рассмотрена в более общем виде в лемме 3. Случаи $\delta=3$ и $\delta \leqslant 1$ пока не изучены. Заметим также, что при таком условии $R=1$. К этому случаю легко свести вариант $b_{k} \asymp k^{-\delta} / R^{k}$. Для этого достаточно рассмотреть обобщенную схему размещения, в которой вероятности (1) определяются последовательностью $b^{\prime}=\left(b_{0}, b_{1} R, b_{2} R^{2}, \ldots\right)$.

Лемма 4. Пусть $N \rightarrow \infty, b_{k} \asymp k^{-\delta}, 1<\delta<3, x \rightarrow 1$ таким образом, что $\mu_{4} /\left(\sigma^{4} N\right) \rightarrow 0$. Пусть $r \asymp N a$. Тогда равномерно относительно чельх $h$

$$
\sigma \sqrt{N} \mathbf{P}\left\{Z_{N}=h\right\}-\frac{1}{\sqrt{2 \pi}} \exp \left\{-\frac{(h-N a)^{2}}{2 \sigma^{2} N}\right\} \rightarrow 0 .
$$

Доказательство. По лемме 2 распределение $Z_{N}$ слабо сходится к нормальному закону. Для доказательства локальной сходимости воспользуемся теоремой 1 из [6], где требуется выполнение следующего условия: $H_{N} \rightarrow \infty$ и существуют такие $v<1 / 2, \alpha>0$ и $\rho \in(0,2]$, что для $u \in\left[H_{N}^{\nu}, \sigma \sqrt{N}\right]$

$$
E_{N}^{2}(u) \geqslant \alpha(\sigma \sqrt{N})^{\rho} u^{2-\rho}
$$

Ниже будет доказано, что данное неравенство выполнено при $\rho=\delta-1$ для любого $v \in(0,1 / 2)$.

Величина $H_{N}$ оценивается снизу точно так же, как при доказательстве леммы 3 , поэтому $H_{N} \rightarrow \infty$.

Пусть $u \in\left[A_{1} \sqrt{\mu_{4}} / \sigma, \sigma \sqrt{N}\right]$, постоянная $A_{1}$ будет определена ниже. Из неравенства Гельдера нетрудно получить, что

$$
\mathbf{E} \zeta_{1} \leqslant\left(\mathbf{E}\left(\zeta_{1}\right)^{4}\right)^{1 / 4}, \quad \mathbf{E}\left(\zeta_{1}\right)^{2} \leqslant\left(\mathbf{E}\left(\zeta_{1}\right)^{4}\right)^{1 / 2}, \quad \mathbf{E}\left(\zeta_{1}\right)^{3} \leqslant\left(\mathbf{E}\left(\zeta_{1}\right)^{4}\right)^{3 / 4}
$$


Используя эти неравенства, получаем следующую оценку:

$$
\begin{aligned}
E_{N}^{2}(u) & =2 N \sum_{0 \leqslant k \leqslant u} k^{2} \mathbf{P}\left\{\zeta_{1}-\zeta_{2}=k\right\}=2 N\left((1 / 2) \mathbf{D}\left(\zeta_{1}-\zeta_{2}\right)-\sum_{k>u} k^{2} \mathbf{P}\left\{\zeta_{1}-\zeta_{2}=k\right\}\right) \\
& \geqslant 2 N\left(\mathbf{D} \zeta_{1}-\frac{1}{u^{2}} \sum_{k=0}^{\infty} k^{4} \mathbf{P}\left\{\zeta_{1}-\zeta_{2}=k\right\}\right) \geqslant 2 N\left(\mathbf{D} \zeta_{1}-\frac{1}{2 u^{2}} \mathbf{E}\left(\zeta_{1}-\zeta_{2}\right)^{4}\right) \\
& \geqslant 2 N\left(\mathbf{D} \zeta_{1}-\frac{8}{u^{2}} \mathbf{E}\left(\zeta_{1}\right)^{4}\right) .
\end{aligned}
$$

Нетрудно видеть, что

$$
\begin{gathered}
a^{2} \leqslant \mathbf{E} \xi_{1}^{2}, \quad \mathbf{E} \zeta_{1} \preccurlyeq a, \quad \mathbf{E}\left(\zeta_{1}\right)^{3} \preccurlyeq \mathbf{E} \xi_{1}^{3}, \\
\left|\mathbf{D} \zeta_{1}-\mathbf{D} \xi_{1}\right|=\left|\mathbf{E}\left(\zeta_{1}\right)^{2}-\mathbf{E} \xi_{1}^{2}+a^{2}-\left(\mathbf{E} \zeta_{1}\right)^{2}\right| \\
\leqslant \sum_{k \leqslant r} k^{2} \mathbf{P}\left\{\xi_{1}=k\right\}\left(\frac{1}{1-P_{r}}-1\right)+\sum_{k>r} k^{2} \mathbf{P}\left\{\xi_{1}=k\right\} \\
+\left(a+\mathbf{E} \zeta_{1}\right)\left(\sum_{k \leqslant r} k \mathbf{P}\left\{\xi_{1}=k\right\}\left(\frac{1}{1-P_{r}}-1\right)+\sum_{k>r} k \mathbf{P}\left\{\xi_{1}=k\right\}\right) \\
\preccurlyeq \mathbf{E} \xi_{1}^{2} P_{r}+\mathbf{E} \xi_{1}^{4} /(N a)^{2}+a^{2} P_{r}+\mathbf{E} \xi_{1}^{2} / N,
\end{gathered}
$$

где мы воспользовались условием $r \asymp N a$.

Покажем, что $a^{2}=o\left(\mathrm{E} \xi_{1}^{2}\right)$. Если $a \preccurlyeq 1$, то данное соотношение очевидно, поскольку $\mathbf{E} \xi_{1}^{2} \rightarrow \infty$ при выбранном $\delta$. Пусть $a \rightarrow \infty$. Для $0<\varepsilon<1$ определим $A=A(\varepsilon)$ следующим образом: $A$ есть наименьшее целое такое, что $\sum_{k>A} b_{k} \leqslant \varepsilon$. Из неравенства Гельдера получаем, что

$$
\left(\sum_{k>A} k x^{k} b_{k}\right)^{2} \leqslant \sum_{k>A} x^{k} b_{k} \sum_{k>A} k^{2} x^{k} b_{k} \leqslant \varepsilon \sum_{k>A} k^{2} x^{k} b_{k} .
$$

Суммы

$$
\sum_{k \leqslant A} k x^{k} b_{k}, \quad \sum_{k \leqslant A} k^{2} x^{k} b_{k}
$$

ограничены, поэтому

$$
a=(1+o(1)) \sum_{k>A} k x^{k} b_{k}, \quad \mathbf{E} \xi_{1}^{2}=(1+o(1)) \sum_{k>A} k^{2} x^{k} b_{k}
$$

Отсюда следует, что $\overline{\lim } a^{2} / \mathbf{E} \xi_{1}^{2} \leqslant \varepsilon$ для любого $\varepsilon>0$. Поэтому $a^{2}=o\left(\mathbf{E} \xi_{1}^{2}\right)$ и, следовательно, $\mathbf{E} \xi_{1}^{2} \asymp \sigma^{2}$. Аналогично можно доказать, что $\mu_{4} \asymp \mathbf{E} \xi_{1}^{4}$.

Из условия $\mu_{4} /\left(\sigma^{4} N\right) \rightarrow 0$ и леммы 2 следует, что

$$
\mathbf{E} \xi_{1}^{4} /(N a)^{2} \preccurlyeq \mu_{4} /\left(\sigma^{2} N\right)=o\left(\sigma^{2}\right) .
$$

Поэтому из (12) находим, что $D \zeta_{1}-D \xi_{1}=o\left(\sigma^{2}\right)$. Таким образом, используя (11), получаем, что

$$
E_{N}^{2}(u) \succcurlyeq N\left(\sigma^{2}-\mu_{4} / u^{2}\right) \text {. }
$$


Так как $u \geqslant A_{1} \sqrt{\mu_{4}} / \sigma$, то $\mu_{4} /\left(u^{2} \sigma^{2}\right) \leqslant 1 / A_{1}^{2}$. Поэтому при достаточно большом $A_{1}$ получим, что $E_{N}^{2}(u) \succcurlyeq N \sigma^{2}$ для всех $u \in\left[A_{1} \sqrt{\mu_{4}} / \sigma, \sigma \sqrt{N}\right]$. С другой стороны, для таких $u$ справедливо неравенство $u^{3-\delta}(\sigma \sqrt{N})^{\delta-1} \leqslant N \sigma^{2}$. Так что

$$
E_{N}^{2}(u) \succcurlyeq u^{3-\delta}(\sigma \sqrt{N})^{\delta-1}
$$

для $u \in\left[A_{1} \sqrt{\mu_{4}} / \sigma, \sigma \sqrt{N}\right]$.

Пусть теперь $u \in\left[H_{N}^{v}, A_{1} \sqrt{\mu_{4}} / \sigma\right]$. Заменяя суммирование интегрированием, при $\delta<3$ нетрудно получить, что для $p \geqslant 2$

$$
\sum_{k} k^{p} b_{k} x^{k} \asymp \sum_{k} k^{p-\delta} x^{k} \asymp \frac{1}{(1-x)^{p+1-\delta}} .
$$

Отсюда и из соотношений $\sigma^{2} \asymp \mathbf{E} \xi_{1}^{2}, \mu_{4} \asymp \mathbf{E} \xi_{1}^{4}$ следует, что $\sqrt{\mu_{4}} / \sigma \asymp(1-x)^{-1}$. Поэтому равномерно по $и$ из рассматриваемого отрезка $x^{u} \asymp 1$. Учитывая, что $\sigma \sqrt{N}=o(r)$, получаем оценку

$$
E_{N}^{2}(u) \succcurlyeq N \sum_{0 \leqslant k \leqslant u} k^{2} b_{k} \succcurlyeq N \int_{A}^{u} y^{2-\delta} d y \succcurlyeq N\left(u^{3-\delta}-A^{3-\delta}\right),
$$

где $A$ - такое число, что для $k \geqslant A$ имеет место неравенство $b_{k} \geqslant c k^{-\delta}$, в котором постоянная $c>0$. Однако при $v>0$ для $u>H_{N}^{v}$ получим, что $A^{3-\delta}=o\left(u^{3-\delta}\right)$, откуда

$$
E_{N}^{2}(u) \succcurlyeq N u^{3-\delta}
$$

для $u \in\left[H_{N}^{\nu}, A_{1} \mathrm{E} \xi_{1}^{3} / \sigma^{2}\right]$. Кроме того, $N>(\sigma \sqrt{N})^{\delta-1}$. Действительно,

$$
(\sigma \sqrt{N})^{\delta-1} N^{-1} \asymp(1-x)^{-(3-\delta)(\delta-1) / 2} N^{(\delta-3) / 2},
$$

а последнее выражение стремится к нулю, так как условие $\sigma^{4} N / \mu_{4} \rightarrow \infty$ эквивалентно соотношению $N(1-x)^{\delta-1} \rightarrow \infty$. Отсюда видно, что (13) выполнено и в этом случае. Лемма доказана.

Снова отметим, что, полагая $r=\infty$, аналогично предыдущему можно доказать, что в условиях леммы 4 выполнена локальная сходимость распределения $S_{N}$ к нормальному закону.

Как уже отмечалось, вероятности (3) не зависят от параметра $x$, поэтому его выбор в зависимости от $N, n$ производится так, чтобы было проще получать асимптотику вероятностей $\mathbf{P}\left\{S_{N}=n\right\}$ и $\mathbf{P}\left\{Z_{N}=n\right\}$ с помощью локальных предельных теорем. Доказанные выше леммы 3 и 4 дают асимптотику этих вероятностей для $n$ таких, что $n=N a+O(\sigma \sqrt{N})$. Поэтому для применения этих лемм при получении результатов о поведении максимальной компоненты в обобщенной схеме размещения достаточно выбрать параметр $x$ как корень уравнения $a=n / N$. Дифференцируя $a$ по $x$, нетрудно получить, что $a$ строго возрастает на интервале $(0, R)$. Кроме того, $a$ непрерывно зависит от $x$ и принимает все значения из интервала $\left(0, \mu_{1}\right)$, где $\mu_{1}=\lim _{x \rightarrow R-0} a$. Заметим, что $\mu_{1}$ зависит только от последовательности $b$. Если $\mu_{1}=\infty$, то уравнение $a=n / N$ всегда имеет единственное решение. Если $\mu_{1}<\infty$, то уравнение $a=n / N$ имеет единственное решение при $n / N \leqslant \mu_{1}$ и не имеет решений при $n / N>\mu_{1}$. В дальнейшем, предполагая, что $x$ выбрано так, что $a=n / N$, мы тем самым накладываем ограничение на поведение дроби $n / N$.

Следующие две теоремы дают достаточные условия невозникновения гигантской компоненты в обобщенной схеме размещения. 
Теорема 1. Пусть параметр $x$ определяется из соотношения $a=n / N$, при этом $n, N \rightarrow \infty$ так, что $N x^{m} \rightarrow \infty, \mathbf{E} \xi_{1}^{2} \preccurlyeq 1$. Тогда в обобщенной схеме размещения гигантской компоненты нет.

Доказательство. Пусть $r=[n t], t>0$. Как было отмечено при доказательстве леммы 3 , из условия $N x^{m} \rightarrow \infty$ следует, что $\sigma \sqrt{N} \rightarrow \infty$. Отсюда и из Е $\xi_{1}^{2} \preccurlyeq 1$ по лемме 2 получаем, что $\sigma \sqrt{N}=o(n)$, поэтому

$$
N P_{r}=N \mathbf{P}\left\{\xi_{1}>n t\right\} \leqslant N \mathbf{P}\left\{\xi_{1}>\sigma \sqrt{N}\right\} \leqslant \sigma^{-2} \sum_{k>\sigma \sqrt{N}} k^{2} x^{k} b_{k} / B(x) .
$$

Поэтому $N P_{r} \rightarrow 0$ и $\left(1-P_{r}\right)^{N} \rightarrow 1$. Кроме того, согласно лемме 3 случайные величины $S_{N}$ и $Z_{N}$ имеют одно и то же предельное распределение, следовательно, $\mathbf{P}\left\{Z_{N}=n\right\} / \mathbf{P}\left\{S_{N}=n\right\} \rightarrow 1$.

Отсюда и из равенства (5) получаем, что при каждом $t>0$ выполняется предельное соотношение $\mathbf{P}\left\{\eta_{(N)} \leqslant n t\right\} \rightarrow 1$, то есть гигантская компонента не возникает.

Аналогично, с помощью леммы 4 доказывается следующее утверждение.

Теорема 2. Пусть $b_{k} \asymp k^{-\delta}, 1<\delta<3$, параметр $x$ определяется из соотношения $a=n / N, n, N \rightarrow \infty$ так, что $N x^{m} \rightarrow \infty u N(1-x)^{\delta-1} \rightarrow \infty$. Тогда в обобщенной схеме размещения гигантской компоненты нет.

Доказательство. Если $x \neq 1-\varepsilon$, где $\varepsilon$ произвольно малое фиксированное положительное число, то $\mathbf{E} \xi_{1}^{2} \preccurlyeq 1$, и выполнены условия теоремы 1 .

Пусть $x \rightarrow 1$. Как уже было отмечено при доказательстве леммы 4, условие $N(1-x)^{\delta-1} \rightarrow \infty$ эквивалентно условию $\mu_{4} /\left(\sigma^{4} N\right) \rightarrow 0$. Поэтому из леммы 2 следует, что $\sigma \sqrt{N}=o(n)$. Кроме того, $a=o(\sigma \sqrt{N})$, поскольку, как было показано при доказательстве леммы $4, a^{2}=o\left(\sigma^{2}\right)$. Следовательно,

$$
N P_{r}=N \mathbf{P}\left\{\xi_{1}>n t\right\} \leqslant N \mathbf{P}\left\{\left|\xi_{1}-a\right|>\sigma \sqrt{N}\right\} \leqslant \mu_{4} /\left(\sigma^{4} N\right) \rightarrow 0 .
$$

Итак, $\left(1-P_{r}\right)^{N} \rightarrow 1$. Кроме того, в условиях теоремы вероятности $\mathbf{P}\left\{S_{N}=n\right\}$ и $\mathbf{P}\left\{Z_{N}=n\right\}$ имеют одну и ту же асимптотику по лемме 4. Из равенства (5) получаем, что при каждом $t>0$ выполняется предельное соотношение $\mathbf{P}\left\{\eta_{(N)} \leqslant n t\right\} \rightarrow 1$. Поэтому гигантская компонента в обобщенной схеме размещения отсутствует.

Следует отметить, что доказанные теоремы в большинстве случаев не дают исчерпывающего ответа на вопрос о возникновении или отсутствии гигантской компоненты в обобщенной схеме размещения. В приведенных ниже примерах только в случае конечного носителя $b$ (пример 2) полученные в статъе результаты доказывают отсутствие гигантской компоненты. Теоремы 1 и 2 полезны тем, что указывают зону изменения параметров, в которой гигантская компонента не возникает, и тем самым сужают область ее поиска.

Рассмотрим несколько примеров применения теорем 1 и 2.

1. Классическая схема размещения. Рассмотрим равновероятное размещение $n$ частиц по $N$ ячейкам. Пусть $\eta_{j}$ - число частиц, попавших в ячейку с номером $j, j=1, \ldots, N$. В этом случае вероятности (1) имеют вид

$$
\mathbf{P}\left\{\eta_{1}=n_{1}, \ldots, \eta_{N}=n_{N}\right\}=\frac{n !}{n_{1} ! \ldots n_{N} ! N^{n}} .
$$


Данная обобщенная схема размещения достаточно хорошо изучена [2]. Известно, что в ней гигантская компонента не возникает при любом поведении дроби $n / N$ и при $N \rightarrow \infty$.

Нетрудно проверить, что равенство (14) выполняется при $b_{k}=1 / k$ !. При этом $\xi_{1}$ имеет распределение Пуассона с параметром $x$, а радиус сходимости $R$ ряда $\sum_{k} x^{k} b_{k}$ равен $\infty$. Заметим также, что для данных $b_{k}$ имеем $k_{1}=m=1$. Выбирая $x$ так, что $a=x=n / N$, по теореме 1 получаем, что для $n / N \preccurlyeq 1$ в классической схеме размещения гигантская компонента не возникает.

2. Предположим, что носитель $b$ конечен. Как нетрудно проверить, в этом случае $R=\infty$ и $\mathbf{E} \xi_{1}^{2} \preccurlyeq 1$. Отсюда следует, что при условии $N x^{m} \rightarrow \infty$, где $x$ выбрано так, что $a=n / N$, по теореме 1 в обобщенной схеме размещения нет гигантской компоненты.

Рассмотрим пример 1.3.2 книги [1]. Из урны, содержащей $l$ шаров каждого из $N$ цветов, с помощью случайного выбора без возвращения извлекаются $n$ шаров. Обозначим $\eta_{i}$ число извлеченных шаров $i$-го цвета, $i=1, \ldots, N$. Тогда для неотрицательных целых $n_{1}, \ldots, n_{N}$ таких, что $n_{1}+\ldots+n_{N}=n$,

$$
\mathbf{P}\left\{\eta_{1}=n_{1}, \ldots, \eta_{N}=n_{N}\right\}=\left(\begin{array}{c}
l \\
n_{1}
\end{array}\right) \ldots\left(\begin{array}{c}
l \\
n_{N}
\end{array}\right) /\left(\begin{array}{l}
l \\
n
\end{array}\right) \text {. }
$$

Равенство (3) выполнено, если

$$
\mathbf{P}\left\{\xi_{1}=k\right\}=\left(\begin{array}{l}
l \\
k
\end{array}\right) p^{k}(1-p)^{l-k}=\left(\begin{array}{l}
l \\
k
\end{array}\right) x^{k} / B(x), \quad k=0,1, \ldots, l,
$$

где $x=p /(1-p), B(x)=(1+x)^{l}$. Поскольку $a=l p$, из уравнения $a=n / N$ получаем, что $N p=n / l$. Поэтому при $n \rightarrow \infty$ выполнено условие $N x^{m} \rightarrow \infty$, так как в данном случае $m=1$. Следовательно, в данной обобщенной схеме при $N, n \rightarrow \infty$ и фиксированном $l$ гигантская компонента не возникает.

3. В [7] изучаются леса Гальтона-Ватсона с $N$ корневыми и $n$ некорневыми вершинами. Пусть $\xi$ - случайная величина, равная числу потомков в соответствующем процессе Гальтона-Ватсона,

$$
\mathbf{P}\{\xi=k\}=\lambda^{k} p_{k} / F(\lambda),
$$

где

$$
F(\lambda)=\sum_{k=0}^{\infty} \lambda^{k} p_{k}, \quad F(1)=1, \quad F^{\prime}(1)=1, \quad \lambda F^{\prime}(\lambda) / F(\lambda)=n /(N+n) .
$$

Пусть $d$ - максимальный шаг $\xi$. Обозначим также $\eta_{1}, \ldots, \eta_{N}$ объемы деревьев в лесе Гальтона-Ватсона. В [7] доказано, что

$$
\mathbf{P}\left\{\eta_{1}=n_{1}, \ldots, \eta_{N}=n_{N}\right\}=\mathbf{P}\left\{v_{1}=n_{1}, \ldots, v_{N}=n_{N} \mid v=N+n\right\},
$$

где $v_{1}, \ldots, v_{N}$ - независимые одинаково распределенные случайные величины, $v=v_{1}+\ldots+v_{N}$, причем

$$
\mathbf{P}\left\{v_{1}=1+k d\right\}=\lambda^{k d} b_{k} / F^{k d+1}(\lambda), \quad b_{k} \asymp k^{-3 / 2} .
$$

В $[7,8]$ получены результаты, из которых видно, что при условии $n / N^{2} \preccurlyeq 1$ и $N \lambda^{d m} \rightarrow \infty$ гигантская компонента в данной обобщенной схеме размещения не возникает, а при условии $n / N^{2} \rightarrow \infty$ возникает.

Полагая $\xi_{i}=\left(v_{i}-1\right) / d, x=(\lambda / F(\lambda))^{d}$, приходим к обобщенной схеме размещения, удовлетворяющей изложенным в начале статьи требованиям. Из теоремы 2 следует, что 
при выполнении условий $N x^{m} \rightarrow \infty, N^{2}(1-x) \rightarrow \infty$ в лесе Гальтона-Ватсона гигантское дерево не возникает [8].

Заметим также, что при $x \rightarrow 1$ имеем $1-x \asymp 1-x^{1 / d}$ и, кроме того, нетрудно доказать, что $1-\lambda F^{\prime}(\lambda) / F(\lambda) \asymp(1-\lambda)^{2}$. Как показано в [7], $1-\lambda \asymp N / n$, поэтому условие $N^{2}(1-x) \rightarrow \infty$ эквивалентно условию $n / N^{2} \rightarrow 0$ (см. [7, 8]).

4. Рассмотрим множество всех лесов из $N$ некорневых деревьев с $n$ вершинами. Зададим на нем равномерное распределение. Обозначим $\eta_{1}, \ldots, \eta_{N}$ размеры деревьев в полученном случайном лесе. Тогда, как показано в [3], для изучения вероятностей $\mathbf{P}\left\{\eta_{1}=n_{1}, \ldots, \eta_{N}=n_{N}\right\}$ применима обобщенная схема размещения, в которой

$$
\mathbf{P}\left\{\xi_{1}=k\right\}=\frac{k^{k-2} x^{k}}{k ! B(x)}, \quad k=1,2, \ldots,
$$

т.е. $b_{k}=k^{k-2} / k$ !. Радиус сходимости $B(x)$ равен $e^{-1}$. Нетрудно видеть, что $b_{k} \asymp k^{-5 / 2} / R^{k}$.

В книге [3] приводятся теоремы, из которых видно, что при выполнении условий

$$
N \theta \rightarrow \infty, \quad N(1-\theta)^{3} \rightarrow \infty, \quad \theta=2(n-N) / n,
$$

гигантская компонента не возникает. Однако при условии $N(1-2(n-N) / n)^{3} \asymp 1$, как показано в [3], в данной обобщенной схеме размещения гигантская компонента все еще отсутствует, а появляется лишь при $N(1-2(n-N) / n)^{3} \rightarrow-\infty$.

Чтобы перейти к обобщенной схеме размещения с такими параметрами, которые удовлетворяют условиям теоремы 2, рассмотрим независимые одинаково распределенные случайные величины $\xi_{i}^{\prime}, i=1, \ldots, N$, с распределением

$$
\mathbf{P}\left\{\xi_{1}^{\prime}=k\right\}=y^{k} b_{k}^{\prime} / \beta(y), \quad k=0,1, \ldots,
$$

где

$$
y=x e, \quad b_{k}^{\prime}=(k+1)^{k-1} /\left((k+1) ! e^{k} B(1 / e)\right), \quad \beta(y)=\sum_{k=0}^{n} y^{k} b_{k}^{\prime} .
$$

Находя асимптотику $b_{k}^{\prime}$ с помощью формулы Стирлинга, получаем, что $b_{k}^{\prime} \asymp k^{-5 / 2}$ при $k \rightarrow \infty$. По теореме 2 при выполнении условий

$$
N y \rightarrow \infty, \quad N(1-y)^{3 / 2} \rightarrow \infty
$$

в рассматриваемой обобщенной схеме гигантской компоненты нет. Следуя обозначениям книги [3], положим $\theta e^{-\theta}=x=y / e, 0<\theta \leqslant 1$. При этом равенство $\mathbf{E} \xi_{1}^{\prime}=n / N$ имеет место при $\theta=2(n-N) / n$. Заметим, что это равенство выполняется только при $n / N \leqslant 2$. Для другой зоны изменения параметров $N, n$ теорема 2 неприменима.

При $y \rightarrow 1$ получим, что $\theta \rightarrow 1$ и

$$
1-y=1-\theta e^{1-\theta}=1-\theta\left(1+(1-\theta)+\frac{(1-\theta)^{2}}{2}+o(1-\theta)^{2}\right)=\frac{(1-\theta)^{2}}{2}(1+o(1)) \text {. }
$$

Отсюда следует, что условия (16) эквивалентны (15). 


\section{Список литературы}

1. Колчин В. Ф., Случайные отображения. Наука, Москва, 1984.

2. Колчин В. Ф., Севастьянов Б. А., Чистяков В. П., Случайные размещения. Наука, Москва, 1976.

3. Колчин В. Ф., Случайные графы. Наука, Москва, 2000.

4. Колчин В. Ф., О существовании гигантской компоненты в схемах размещения частиц. Обозрение прикладной и промымленной математики (2000) 7, №1, 112-113.

5. Грэхем Р., Кнут Д., Паташник О., Конкретная математика. Мир, Москва, 1998.

6. Мухин А. Б., Локальные предельные теоремы для решетчатых случайных величин. Теория вероятностей и ее применения (1991) 36, №4, 660-674.

7. Pavlov Yu. L., Random Forests. VSP, Utrecht, 2000.

8. Чеплюкова И. А., Возникновение гигантского дерева в случайном лесе. Дискретная математика (1998) 10, №1, 111-126.

Статья поступила 17.01.2002. 